\title{
The Vorticity Equations in Orthogonal \\ Curvilinear Coordinates \\ by
}

\section{H. Arakawa}

Meteorological Research Institute

(Received July 28,1950 )

\begin{abstract}
The transformation of the equations of motion of a viscous fluid to orthogonal curvilinear coordinates has been discussed by G. B. JEFFERY, and that of the equations of motion in dynamical meteorolocy to orthogonal curvilinear coordinates by the present author, sut the analogous equations for the vorticity do not appear to have attracted the same attention. The first section of this paper deals with the transformation of the vorticity equations to the orthogonal curvilinear coordinates. The second section is devoted to applications to cylindrical and spherical polar coordinates.
\end{abstract}

\section{Transformation of the Vorticity Fquation}

If $V$ be the velocity, $K$ the externally applied force, $p$ the pressure, $\rho$ the density, $W$ the angular velocity of the earth's rotation, then the eqation of motion in vectorial form is

or

$$
\frac{d V}{d t}+2[\boldsymbol{W} \times \boldsymbol{V}]=K-\frac{1}{\rho} \nabla p
$$

$$
\frac{\partial \boldsymbol{V}}{\partial \boldsymbol{t}}+(\boldsymbol{V} \cdot \boldsymbol{\nabla}) \boldsymbol{V}+2[\boldsymbol{W} \times \boldsymbol{V}]=\boldsymbol{K}-\frac{1}{\rho} \nabla \boldsymbol{p} .
$$

Taking the operation CURL on both sides of the above equation, and using the relations

$$
\begin{aligned}
& \operatorname{rot}\left(\frac{\partial \boldsymbol{V}}{\partial t}\right)=\frac{\partial}{\partial t}(\operatorname{rot} \boldsymbol{V}), \\
& \operatorname{rot}\{(\boldsymbol{V} \cdot \nabla) \boldsymbol{V}\}=(\boldsymbol{V} \cdot \nabla) \operatorname{rot} \boldsymbol{V}-(\operatorname{rot} \boldsymbol{V} \cdot \boldsymbol{\nabla}) \boldsymbol{V}+\operatorname{rot} \boldsymbol{V} \cdot \operatorname{div} \boldsymbol{V}, \\
& \operatorname{rot}[\boldsymbol{W} \times \boldsymbol{V}]=(\boldsymbol{V} \cdot \nabla) \boldsymbol{W}-(\boldsymbol{W} \cdot \boldsymbol{\nabla}) \boldsymbol{V}+\boldsymbol{W} \cdot \operatorname{div} \boldsymbol{V}-\boldsymbol{V} \cdot \operatorname{div} \boldsymbol{W}, \\
& \operatorname{rot}\left(\frac{1}{\rho} \nabla p\right)=-\frac{1}{\rho^{2}}(\nabla \rho \times \nabla p), \\
& \operatorname{div} \boldsymbol{W}=0,
\end{aligned}
$$

we get the vorticity equation as :

$$
\underset{\partial t}{\partial(\operatorname{rot} V)}+(\boldsymbol{V} \cdot \boldsymbol{\nabla})(\operatorname{rot} \boldsymbol{V}+2 \boldsymbol{W})+(\operatorname{rot} \boldsymbol{V}+2 \boldsymbol{W}) \operatorname{div} \boldsymbol{V}
$$


Again using the relations

$$
-\{(\operatorname{rot} V+2 W) \cdot \nabla\} V=\operatorname{rot} K+\frac{1}{\rho^{2}}(\nabla \rho \times \nabla p) .
$$

$$
\begin{gathered}
\operatorname{rot}\{(\operatorname{rot} \boldsymbol{V}+2 \boldsymbol{W}) \times \boldsymbol{V}\}=(\boldsymbol{V} \cdot \boldsymbol{\nabla})(\operatorname{rot} \boldsymbol{V}+2 \boldsymbol{W})-\{(\operatorname{rot} \boldsymbol{V}+2 \boldsymbol{W}) \cdot \boldsymbol{\nabla}\} \boldsymbol{V} \\
+(\operatorname{rot} \boldsymbol{V}+2 \boldsymbol{W}) \cdot \operatorname{div} \boldsymbol{V}-\boldsymbol{V} \cdot \operatorname{div}(\operatorname{rot} \boldsymbol{V}+2 \boldsymbol{W}),
\end{gathered}
$$

and

$$
\frac{\partial W}{\partial t}=0, \quad \operatorname{div} W=0, \quad \operatorname{div} \cdot \operatorname{rot} V=0,
$$

we get the vorticity equation as follows :

$$
\frac{\partial(\operatorname{rot} V+2 W)}{\partial t}+\operatorname{rot}\{(\operatorname{rot} V+2 W) \times V\}=\operatorname{rot} \boldsymbol{K}+\frac{1}{\rho^{2}}(\nabla \rho \times \nabla p) \text {. }
$$

The transformations of div and rot follow at once from the definition of these operators in terms of surface and line integrals, respectively.

If $\alpha, \beta, \gamma$ are orthogonal curvilinear coordinates, and if

$$
\begin{array}{ll}
h_{1}{ }^{2}=\left(\frac{\partial \alpha}{\partial x}\right)^{2}+\left(\frac{\partial \alpha}{\partial y}\right)^{2}+\left(\frac{\partial \alpha}{\partial z}\right)^{2}, & h_{2}{ }^{2}=\left(\frac{\partial \beta}{\partial x}\right)^{2}+\left(\frac{c \beta}{\partial y}\right)^{2}+\left(\frac{\partial \beta}{\partial z}\right)^{2}, \\
h_{s}{ }^{2}=\left(\frac{\partial \gamma}{\partial x}\right)^{2}+\left(\frac{\partial \gamma}{\partial y}\right)^{2}+\left(\frac{\partial \gamma}{\partial z}\right)^{2}, &
\end{array}
$$

so that elements of arc measured along the normals to the coordinate surfaces at any point are $\frac{\delta \alpha}{h_{1}}, \frac{\delta \beta}{h_{2}}, \frac{\delta \gamma}{h_{3}}$, then

$$
\begin{gathered}
\operatorname{rot} V\left[\zeta_{1}=h_{2} h_{3}\left\{\frac{\partial}{\partial \beta}\left(\frac{v_{3}}{h_{3}}\right)-\frac{\partial}{\partial \gamma}\left(\frac{v_{2}}{h_{2}}\right)\right\}, \quad \zeta_{2}=h_{3} h_{1}\left\{\frac{\partial}{\partial \gamma}\left(\frac{v_{1}}{h_{1}}\right)-\frac{\partial}{\partial \alpha}\left(\begin{array}{c}
v_{3} \\
h_{3}
\end{array}\right)\right\},\right. \\
\left.\zeta_{3}=h_{1} h_{2}\left\{\frac{\partial}{\partial \alpha}\left(\begin{array}{c}
v_{2} \\
h_{2}
\end{array}\right)-\frac{\partial}{\partial \beta}\left(\begin{array}{c}
v_{1} \\
h_{1}
\end{array}\right)\right\}\right], \\
\operatorname{div} V=h_{1} h_{2} h_{3}\left\{\frac{\partial}{\partial \alpha}\left(\begin{array}{c}
v_{1} \\
h_{2} h_{3}
\end{array}\right)+\frac{\partial}{\partial \beta}\left(\frac{v_{2}}{h_{3} h_{1}}\right)+\frac{\partial}{\partial \gamma}\left(\frac{v_{3}}{h_{1} h_{2}}\right)\right\},
\end{gathered}
$$

where $v_{1}, v_{2}, v_{3}$ are the components of $V$ along the normals to the surfaces $\alpha, \beta$, $\gamma=$ const., respectively, and $\zeta_{1}, \zeta_{2}, \zeta_{3}$ the components of $\operatorname{rot} V$ along the normals $\alpha$, $\beta, \gamma=$ const., respectively.

By a second application of the operations implied in (2), we have, denoting the direction of a component by a suffix,

$$
\begin{aligned}
\operatorname{rot}_{\alpha}\{(\operatorname{rot} V+2 \boldsymbol{W}) \times V\}=h_{2} h_{:[}\left[\frac{\partial}{\partial \beta}\{\right. & \left.\frac{1}{h_{3}}\left[\left(\zeta_{1}+2 \omega_{1}\right) v_{2}-\left(\zeta_{2}+2 \omega_{2}\right) v_{1}\right]\right\} \\
& \left.-\frac{\partial}{\partial \gamma}\left\{\frac{1}{h_{2}}\left[\left(\zeta_{3}+2 \omega_{3}\right) v_{1}-\left(\zeta_{1}+2 \omega_{1}\right) v_{3}\right]\right\}\right] .
\end{aligned}
$$

Using the relations

$$
\begin{aligned}
& \operatorname{div} W=h h_{-} h\left\{\begin{array}{l}
\partial \\
\bar{c} \alpha
\end{array}\left(\frac{\omega_{1}}{h_{2} h_{3}}\right)+\frac{\partial}{\partial \beta}\left(\frac{\omega_{2}}{h_{3} h_{1}}\right)+\frac{\partial}{\partial \gamma}\left(\begin{array}{c}
\omega_{3} \\
h_{1} h_{2}
\end{array}\right)\right\} \equiv 0, \\
& \operatorname{div} \cdot \operatorname{rot} V=h_{1} h_{2} h_{3}\left\{\frac{\partial}{\partial \alpha}\left(\begin{array}{c}
\zeta_{1} \\
h_{2} h_{3}
\end{array}\right)+\frac{\partial}{\partial \beta}\left(\begin{array}{c}
\zeta_{2} \\
h_{3} h_{1}
\end{array}\right)+\frac{\partial}{\partial \gamma}\left(\begin{array}{c}
\zeta_{3} \\
h_{1} h_{2}
\end{array}\right)\right\} \equiv 0,
\end{aligned}
$$

the last relation can be transformed into an expression which while it appears more complex, is in reality simpler in appilcation, namely, 
(4)

$$
\left\{\begin{aligned}
\operatorname{rot} \alpha & \{(\operatorname{rot} V+2 W) \times V\}=\left(v_{1} h_{1} \frac{\partial}{\partial \alpha}+v_{2} h_{2} \frac{\partial}{\partial \beta}+v_{1} h_{3} \frac{\partial}{\partial \gamma}\right)\left(\zeta_{1}+2 \omega_{1}\right) \\
& +\left(\zeta_{1}+2 \omega_{1}\right) h_{1} h_{2} h_{3}\left\{\frac{\partial}{\partial \alpha}\left(\begin{array}{c}
v_{1} \\
h_{2} h_{3}
\end{array}\right)+\frac{\partial}{\partial \beta}\left(\begin{array}{c}
v_{2} \\
h_{3} h_{\mathrm{i}}
\end{array}\right)+\frac{\partial}{\partial \gamma}\left(\frac{v_{3}}{h_{1} h_{2}}\right)\right\} \\
& -\left\{\left(\zeta_{1}+2 \omega_{1}\right) h_{1} \frac{\partial}{\partial \alpha}+\left(\zeta_{2}+2 \omega_{2}\right) h_{2} \frac{\partial}{\partial \beta}+\left(\zeta_{3}+2 \omega_{3}\right) h_{3} \frac{\partial}{\partial \gamma}\right\} v_{1} \\
& -\left\{\left(\zeta_{1}+2 \omega_{1}\right) v_{2}-\left(\zeta_{2}+2 \omega_{2}\right) v_{1}\right\} h_{1} h_{2} \frac{\partial}{\partial \beta}\left(\begin{array}{c}
1 \\
h_{1}^{-}
\end{array}\right)+\left\{\left(\zeta_{3}+2 \omega_{3}\right) v_{1}\right. \\
& \left.-\left(\zeta_{1}+2 \omega_{1}\right) v_{3}\right\} h_{1} h_{3} \frac{\partial}{\partial \gamma}\left(\frac{1}{h_{1}}\right) .
\end{aligned}\right.
$$

Let

$$
\frac{d}{d t} \equiv \frac{\partial}{\partial t}+v_{\alpha} h_{1} \frac{\partial}{\partial \alpha}+v_{2} h_{2} \frac{\partial}{\partial \beta}+v_{i} h_{3} \frac{\partial}{\partial \gamma},
$$

then the $\alpha$ component of (1.a) becomes

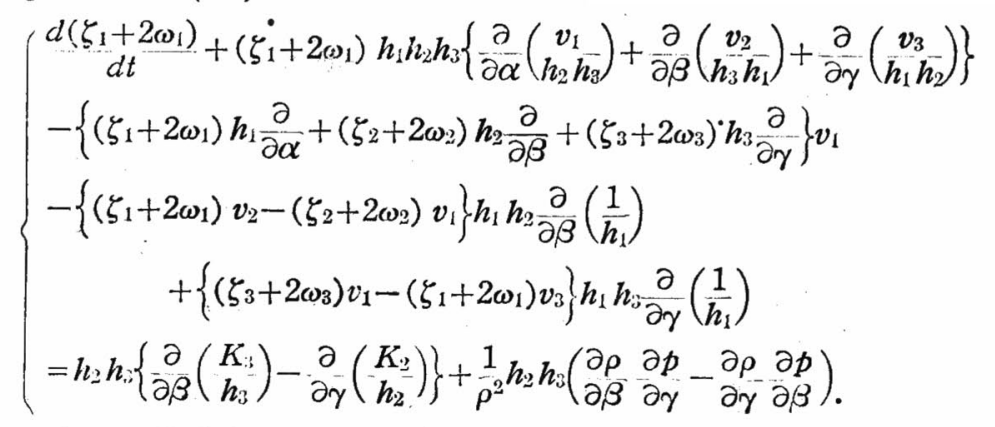

If the externaly applied force $K\left(K_{i}, K_{2}, K_{i}\right)$ has a potential $\varphi$, then using the relation $\operatorname{rot} K=\operatorname{rot} \cdot \operatorname{grad} \rho=0$, the final equation (6) becomes more simple. The corresponding equations in $\zeta_{2}, \zeta_{3}$ can at once be written down from symmetry.

\section{Application to Cylindrical and Spherical Polar Coordinates}

If we take Cartesian coordinates $x, y, z$, with the $x$-axis drawn to south, the $y$-axis drawn to east and the $z$-axis vertically upwards, the component vorticities along these three axes are given by

$$
\zeta_{x}=\frac{\partial v_{z}}{\partial y}-\frac{\partial v_{y}}{\partial z}, \quad \zeta_{y}=\frac{\partial v_{x}}{\partial z}-\frac{\partial v_{z}}{\partial x}, \quad \zeta_{z}=\frac{\partial v_{y}}{\partial x}-\frac{\partial v_{x}}{\partial y},
$$

where $v_{x}, v_{y}, v_{x}$ are the component velocities. The vorticity equations in Cartesian coordinates become

$$
\begin{aligned}
& \frac{d}{d t}\left(\zeta_{x}-2 \omega \cos \emptyset\right)+\left(\zeta_{x}-2 \omega \cos \emptyset\right) \cdot \Theta-\left\{\left(\zeta_{x}-2 \omega \cos \emptyset\right) \frac{\partial v_{x}}{\partial x}\right. \\
&\left.+\zeta_{v} \frac{\partial v_{x}}{\partial y}+\left(\zeta_{z}+2 \omega \sin \emptyset\right) \frac{\partial v_{x}}{\partial z}\right\}=\frac{1}{\rho^{2}}\left(\frac{\partial \rho}{\partial y} \frac{\partial p}{\partial z}-\frac{\partial \rho}{\partial z} \frac{\partial p}{\partial y}\right) \\
& \frac{d}{d t} \zeta_{y}+\zeta_{y} \cdot \Theta-\left\{\left(\zeta_{x}-2 \omega \cos \emptyset\right) \frac{\partial v_{y}}{\partial x}\right. \\
&\left.+\zeta_{y} \frac{\partial v_{y}}{\partial y}+\left(\zeta_{z}+2 \omega \sin \emptyset\right) \frac{\partial v_{y}}{\partial z}\right\}=\frac{1}{\rho^{2}}\left(\frac{\partial \rho}{\partial z} \frac{\partial p}{\partial x}-\frac{\partial \rho}{\partial x} \frac{\partial p}{\partial z}\right) \\
& \frac{d}{d t}\left(\zeta_{z}+2 \omega \sin \emptyset\right)+\left(\zeta_{z}+2 \omega \sin \emptyset\right) \cdot \Theta-\left\{\left(\zeta_{x}-2 \omega \cos \emptyset\right) \frac{\partial v_{z}}{\partial x}\right. \\
&\left.+\zeta_{y} \frac{\partial v_{z}}{\partial y}+\left(\zeta_{z}+2 \omega \sin \emptyset\right) \frac{\partial v_{z}}{\partial z}\right\}=\frac{1}{\rho^{2}}\left(\frac{\partial \rho}{\partial x} \frac{\partial p}{\partial y}-\frac{\partial \rho}{\partial y} \frac{\partial p}{\partial x}\right)
\end{aligned}
$$


where

$$
\frac{d}{d t}=\frac{\partial}{\partial t}+v_{x} \frac{\partial}{\partial x}+v_{y} \frac{\partial}{\partial y}+v_{z} \frac{\partial}{\partial z}
$$

$\emptyset$ is the latitude supposed to be a constant and $\Theta=\operatorname{div} V_{\text {. }}$ These expressions have been given by Th. HESSELBERG and A. FRIEDMANN.

If we take cylindrical coordinates $s, \theta, z$, we have

$$
x=s \cos \theta, y=s \sin \theta, z=z, \quad h_{1}=h_{j}=1 \text { and } h_{2}=\frac{1}{s} .
$$

Then the component vorticities along $s-, \theta-, z$-axis are given by

$$
\zeta_{s}=\frac{1}{s} \frac{\partial v_{s}}{\partial g}-\frac{\partial v_{\theta}}{\partial z}, \quad \zeta_{\theta}=\frac{\partial v_{s}}{\partial z}-\frac{\partial v_{z}}{\partial s}, \quad \zeta_{z}=\frac{\partial v_{\partial}}{\partial s}+\frac{v_{\theta}}{s}-\frac{\partial v_{s}}{s \partial \dot{\theta},}
$$

where $v_{s}, v_{\theta}, v_{t}$ are the component velocities. From (6) the vorticity equations in cylindrical coordinates become

$$
\begin{aligned}
& \frac{d}{d t}\left(\zeta_{s}-2 \omega \cos \emptyset \cos \theta\right)+\left(\zeta_{s}-2 \omega \cos \emptyset \cos \theta\right) \cdot \theta \\
& -\left[\left(\zeta_{s}-2 \omega \cos \emptyset \cos \theta\right) \frac{\partial v_{s}}{\partial s}+\left(\zeta_{\theta}+2 \omega \cos \emptyset \sin \vartheta\right) \frac{\partial v_{s}}{s \partial \theta}+\left(\zeta_{z}+2 \omega \sin \emptyset\right)^{\partial v_{s}} \frac{\partial z}{\partial z}\right] \\
& =\frac{i}{\rho^{2}}\left(\begin{array}{l}
\partial \rho \\
s \partial \theta
\end{array} \frac{\partial p}{\partial z}-\frac{\partial \rho}{\partial z} \frac{\partial p}{s \partial \theta}\right), \\
& \frac{d}{d t}\left(\zeta_{\theta}+2 \omega \cos \oplus \sin \theta\right)+\left(\zeta_{\theta}+2 \omega \cos \phi \sin \theta\right) \cdot \theta \\
& -\left[\left(\zeta_{s}-2 \omega \cos \theta \cos \theta\right) \frac{\partial v_{\partial}}{\partial s}+\left(\zeta_{\theta}+2 \omega \cos \theta \sin \theta\right) \frac{\partial v_{\theta}}{s \partial \theta}+\left(\zeta_{z}+2 \omega \sin \phi\right) \frac{\partial v_{\theta}}{\partial z}\right]
\end{aligned}
$$

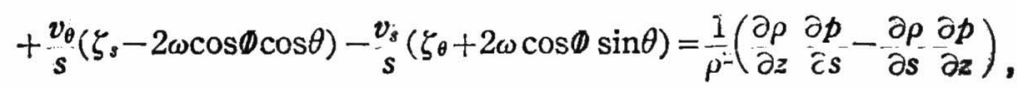

$$
\begin{aligned}
& \frac{d}{d t}\left(\zeta_{z}+2 \omega \sin \theta\right)+\left(\zeta_{z}+2 \omega \sin \theta\right) \cdot \Theta \\
& -\left[\left(\zeta_{s}-2 \omega \cos \theta \cos \theta\right) \frac{\partial v_{z}}{\partial s}+\left(\zeta_{\theta}+2 \omega \cos \theta \sin \theta\right) \frac{\partial v_{z}}{s} \partial \theta+\left(\zeta_{z}+2 \omega \sin \theta\right) \frac{\partial v_{z}}{\partial z}\right] \\
& =\frac{1}{\rho^{i}}\left(\frac{\partial \rho}{\partial s} \frac{\partial p}{s} \partial \theta-\frac{\partial \rho}{s \partial \theta} \partial \dot{\partial}\right),
\end{aligned}
$$

where

$$
\begin{aligned}
\frac{d}{d t} & =\frac{\partial}{\partial t}+v_{s} \frac{\partial}{\partial s}+v_{\theta} \frac{\partial}{s \partial \theta}+v_{z} \frac{\partial}{\partial z}, \\
\Theta & =\frac{1}{s} \frac{\partial}{\partial s}\left(s v_{s}\right)+\frac{1}{s} \frac{\partial v_{\theta}}{\partial \theta}+\frac{\partial v_{z}}{\partial z,}
\end{aligned}
$$

and $\zeta_{8}-2 \omega \cos \Phi \cos \theta, \zeta_{\theta}+2 \omega \cos \theta_{\sin } \theta, \quad \zeta_{z}+2 \omega \sin \theta$, are the components of absolute vorticity alung $s-, \theta-\underset{(5)}{z \text {-axis, }}$, respectively. These expressions have been discussed by the present author.

If $r, \theta, \lambda$ are the spherical polar coordinates with the geocentric colatitude $\theta$ and Iongitucle $\lambda$, then $h_{1}=1, h_{2}=\frac{1}{r}, h_{3}=\frac{1}{r \sin \theta}$. The component vorticities along $r$, $A_{-}, A-a x i s$ are given by

$$
\zeta_{r}=\frac{1}{r \sin \theta}\left\{\frac{\partial}{\partial \theta}\left(\sin \theta \cdot v_{\lambda}\right)-\frac{\partial v_{\theta}}{\partial \lambda}\right\}
$$




$$
\begin{aligned}
& \zeta_{\theta}=\frac{1}{r}\left\{\frac{1}{\sin \theta} \frac{\partial v_{r}}{\partial \lambda}-\frac{\partial}{\partial r}\left(r v_{\lambda}\right)\right\}, \\
& \zeta_{\lambda}=\frac{1}{r}\left\{\frac{\partial\left(r \cdot v_{\theta}\right)}{\partial r}-\frac{\partial v_{r}}{\partial \theta}\right\},
\end{aligned}
$$

where $v_{r}, v_{\theta}, v_{\lambda}$ are the component velocities. From (6), the vorticity equations in spherical folar coordinates become

$$
\begin{aligned}
& \frac{d}{d t}\left(\zeta_{r}+2 \omega \cos \theta\right)+\left(\zeta_{r}+2 \omega \cos \theta\right) \cdot \Theta \\
& -\left\{\left(\zeta_{r}+2 \omega \cos \theta\right) \frac{\partial v_{r}}{\partial r}+\left(\zeta_{\theta}-2 \omega \sin \theta\right) \frac{\partial v_{r}}{r \partial \theta}+\zeta_{\lambda} \frac{\partial v_{r}}{r \sin \theta \partial)_{2}}\right\} \\
& =\frac{1}{\rho^{2}}\left(\begin{array}{cccc}
\partial \rho & \partial p & \partial \rho & r \sin \theta \partial \lambda
\end{array}-\frac{\partial \rho}{r \sin 9 \partial \lambda} \frac{\partial p}{r \partial \theta}\right), \\
& \frac{d}{d t}\left(\zeta_{\theta}-2 \omega \sin \theta\right)+\left(\zeta_{\theta}-2 \omega \sin \theta\right) \cdot \Theta \\
& -\left\{\left(\zeta_{r}+2 \omega \cos \theta\right) \frac{\partial v_{\theta}}{\partial r}+\left(\zeta_{\theta}-2 \omega \sin \theta\right) \frac{\partial v_{\theta}}{r} \partial \theta+\zeta_{\lambda} \frac{\partial v_{\theta}}{r \sin 9 \partial \lambda}\right\} \\
& -\frac{v_{r}}{r}\left(\zeta_{\theta}-2 \omega \sin \theta\right)+\frac{v_{\theta}}{r}\left(\zeta_{r}+2 \omega \cos \theta\right) \\
& =\frac{1}{\rho^{2}}\left(\frac{\partial \rho}{r \sin \theta \partial \lambda} \frac{\partial p}{\partial r}-\frac{\partial \rho}{\partial r} r \sin \theta \partial \lambda\right), \\
& \frac{d \zeta_{\lambda}}{d t}+\zeta_{\lambda} \theta-\left\{\left(\zeta_{r}+2 \omega \cos \theta\right) \frac{\partial v_{\lambda}}{\partial r}+\left(\zeta_{\theta}-2 \omega \sin \theta\right) \frac{\partial v_{\lambda}}{r \partial \theta}+\zeta_{\lambda} \frac{\partial v_{\lambda}}{r \sin \theta \partial \lambda}\right\} \\
& -{ }_{r}^{v_{r}} \zeta_{\lambda}+\frac{v_{\lambda}}{r}\left(\zeta_{r}+2 \omega \cos \theta\right)+\frac{\cot \theta_{r}}{v_{\lambda}\left(\zeta_{\theta}-2 \omega \sin \theta\right)-\frac{\cot \theta}{r} v_{\theta} \cdot \zeta_{\lambda}} \\
& =\frac{1}{\rho^{2}}\left(\frac{\partial \rho}{\partial r} \underset{r \partial \theta}{r}-\frac{\partial \rho}{r \partial \theta} \frac{\partial p}{\partial r}\right) \text {, }
\end{aligned}
$$

where

$$
\begin{aligned}
& \frac{d}{d t}=\frac{\partial}{\partial t}+v_{r} \frac{\partial}{\partial r}+\frac{v_{\theta}}{r} \frac{\partial}{\partial \theta}+\frac{\dot{v_{\lambda}}}{r \sin \theta} \frac{\partial}{\partial \lambda} \text {. } \\
& \Theta=\frac{1}{r^{2}} \frac{\partial}{\partial r}\left(r^{2} v_{r}\right)+\frac{1}{r \sin \theta} \partial_{\theta}\left(v_{\theta} \sin \theta\right)+\frac{1}{r \sin \theta} \frac{\partial v_{\lambda}}{\partial \lambda^{-}},
\end{aligned}
$$

and $\zeta_{r}+2 \omega \cos \theta, \zeta_{\theta}-2 \omega \sin \theta, \zeta_{\lambda}$ are the components of absolute vorticity along $\gamma_{-}, \theta_{-}, \lambda_{-}$ axis, respectively. These expresseions have been discussed by the present author.

\section{Reference}

1). G. B. J.FFERY: The Equations of Motion of a Viscous Fluid, Phil. Mag., Ser. 6, Vol. 29, pp. $445-455$ (1915).

2) H. ARAKAWA: Transformation of the Equations of Motion in Dynamical Meteorology to Orthogonal Curvilinear Coordinates, Papers in Meteorology and Geophysics, Vol. 1, 45-49 (1950).

3). H. ARAKAWA: Lie Wirbelgleichungen mit Berücksichtigung der Frddrehung, Meteorol. Zeitschr., 1941, S. 70-71.

4) Th. HïSSELBERG und A. FRIEDMANN: Die Grössenordnung der meteorologischen Flemente und ihrer räumlichen und zeitlichen Ableitungen, Veröff. Geophys. Inst Univ. Leipzig, Bd. 1, S. 147-173, 1914.

5) H. ARAKAWA: The Vorticity Equations in the Spherical and Cylindrical Coordinates; Geophys. Mag., Tokyo, Vol. 16, pp. 1-4 (1948). 